TRANSACTIONS OF THE

AMERICAN MATHEMATICAL SOCIETY

Volume 353, Number 4, Pages 1635-1659

S 0002-9947(00)02665-9

Article electronically published on November 21, 2000

\title{
ON THE LACK OF NULL-CONTROLLABILITY OF THE HEAT EQUATION ON THE HALF-LINE
}

\author{
SORIN MICU AND ENRIQUE ZUAZUA
}

\begin{abstract}
We consider the linear heat equation on the half-line with a Dirichlet boundary control. We analyze the null-controllability problem. More precisely, we study the class of initial data that may be driven to zero in finite time by means of an appropriate choice of the $L^{2}$ boundary control. We rewrite the system on the similarity variables that are a common tool when analyzing asymptotic problems. Next, the control problem is reduced to a moment problem which turns out to be critical since it concerns the family of real exponentials $\left\{e^{j t}\right\}_{j \geq 1}$ in which the usual summability condition on the inverses of the eigenvalues does not hold. Roughly speaking, we prove that controllable data have Fourier coefficients that grow exponentially for large frequencies. This result is in contrast with the existing ones for bounded domains that guarantee that every initial datum belonging to a Sobolev space of negative order may be driven to zero in an arbitrarily small time.
\end{abstract}

\section{Introduction. Problem formulation}

Given $T>0$, we consider the linear heat equation on the half-line:

$$
\begin{cases}u_{t}(x, t)-u_{x x}(x, t)=0 & \text { for } x \in \Omega, 0<t<T, \\ u(0, t)=v(t) & \text { for } 0<t<T, \\ u(x, 0)=u_{0}(x) & \text { for } x \in \Omega,\end{cases}
$$

where $\Omega=(0, \infty)$.

In (1.1), $v \in L^{2}(0, T)$ is a boundary control and $u=u(x, t)$ is the state.

System (1.1) is said to be null-controllable at time $T$ if for any $u_{0} \in L^{2}(\Omega)$ there exists a control $v \in L^{2}(0, T)$ such that the solution of (1.1) satisfies

$$
u(x, T)=0 \text { for } x>0 .
$$

Notice that, unless some growth conditions are imposed at infinity, equation (1.1) has multiple smooth solutions (see 13, pp. 171-176). We shall define by transposition a class of solutions which do not grow very rapidly at infinity. In this way the uniqueness is ensured.

When $\Omega$ is a bounded interval it is well-known that system (1.1) is null-controllable for any $T>0$. We refer to D. L. Russell [22] for some particular examples treated by means of moment problems and Fourier series, and to A. Fursikov and O. Yu. Imanuvilov 9 and G. Lebeau and L. Robbiano 18 for the general result

Received by the editors July 21, 1999 and, in revised form, October 22, 1999.

2000 Mathematics Subject Classification. Primary 35B37, 35K05.

Key words and phrases. Heat equation, similarity variables, control, moments.

The first author was supported by grants PB96-0663 and 303/1999 of CNCSU (Romania).

The second author was supported by grant PB96-0663 of the DGES (Spain). 
in the multi-dimensional case covering any bounded smooth domain $\Omega$. Both the approaches of [9] and [18] are based on the use of Carleman inequalities.

None of the approaches mentioned above apply when $\Omega$ is an unbounded domain.

This paper is devoted to analyzing the particular case in which $\Omega$ is a half-line: $\Omega=\mathbb{R}_{+}=(0, \infty)$.

As we shall see, the situation is completely different to the one we have described above and, roughly speaking, one may say that there is no initial data in any negative Sobolev space that may be driven to zero in finite time. Therefore, in some sense, the situation is the opposite to the one we encounter in the case of bounded domains.

There is a weaker notion of controllability, the so-called approximate controllability property. System (1.1) is said to be approximately controllable in time $T$ if for any $u_{0} \in L^{2}(\Omega)$ the set of reachable states, $R\left(T ; u_{0}\right)=\{u(T): u$ solution of (1.1) with $\left.v \in L^{2}(0, T)\right\}$, is dense in $L^{2}(\Omega)$.

With the aid of classical backward uniqueness results for the heat equation (see, for instance, J. L. Lions and E. Malgrange [17 and J. M. Ghidaglia 10]), it can be seen that null-controllability implies approximate controllability. However one can easily prove the approximate controllability directly, for both bounded and unbounded domains. In fact, using the Hahn-Banach Theorem, it can be seen that approximate controllability is equivalent to the following unique continuation property: If $\varphi=\varphi(x, t)$ solves

$$
\begin{cases}\varphi_{t}(x, t)+\varphi_{x x}(x, t)=0 & \text { for } x \in \Omega, t>0 \\ \varphi(0, t)=0 & \text { for } t>0\end{cases}
$$

and, moreover, $\varphi_{x}(0, t)=0$ for $t>0$, then, necessarily, $\varphi \equiv 0$.

It is easy to see that this uniqueness property holds as a consequence of Holmgren's Uniqueness Theorem. In fact, in view of the infinite speed of propagation underlying the heat equation, this property holds for any domain $\Omega$ (bounded or not) and for any $T>0$.

Thus, since approximate controllability holds, it is natural to analyze whether null-controllability holds as well.

The null-controllability of (1.1) with initial data in $L^{2}\left(\mathbb{R}_{+}\right)$and boundary control in $L^{2}(0, T)$ is equivalent to an observability inequality for the adjoint system

$$
\begin{cases}\varphi_{t}+\varphi_{x x}=0 & \text { for } x \in \Omega, t>0 \\ \varphi=0 & \text { for } x=0, t>0\end{cases}
$$

More precisely, it is equivalent to the existence of a positive constant $C>0$ such that the inequality

$$
\|\varphi(0)\|_{L^{2}\left(\mathbb{R}_{+}\right)}^{2} \leq C \int_{0}^{T}\left|\varphi_{x}(0, t)\right|^{2} d t
$$

holds for every smooth solution of (1.3).

When $\Omega$ is bounded, Carleman inequalities provide the estimate (1.4) and, consequently, null-controllability holds (see for instance [9, [7]).

When $\Omega$ is the half-line it is easy to see that (1.4) may not hold. Indeed, let $\varphi^{0} \in \mathcal{D}\left(\mathbb{R}_{+}\right)$and $\varphi_{k}^{0}(x)=\varphi(x-k)$ with $k>0$ large enough. Let $\varphi_{k}$ be the solution of (1.3) with initial datum $\varphi_{k}^{0}$ at time $t=T$, i.e.

$$
\varphi_{k}(T)=\varphi_{k}^{0} \text { in } \mathbb{R}_{+} .
$$


It is easy to see that

$$
\left\|\varphi_{k}(0)\right\|_{L^{2}\left(\mathbb{R}_{+}^{n}\right)}^{2} / \int_{0}^{T}\left|\left(\varphi_{k}\right)_{x}(0, t)\right|^{2} d t \rightarrow \infty, \text { as } k \rightarrow \infty .
$$

Thus, we may not expect the null controllability result of the case where $\Omega$ is bounded to be true in the case where $\Omega=\mathbb{R}_{+}$.

We refer to L. Rosier [21] for a similar negative result in the context of exact controllability of linear, constant coefficient pde's in one space dimension.

This translation invariance argument does not allow one to exclude weighted versions of the inequality (1.4), and therefore, the null-controllability of suitable initial data $u_{0}$ may not be excluded. Nevertheless, let us remark that our arguments show that there is no positive weight $\rho$ such that the inequality

$$
\int_{0}^{\infty}|\varphi(x, 0)|^{2} \rho(x) d x \leq C \int_{0}^{T}\left|\varphi_{x}(0, t)\right|^{2} d t
$$

holds for every smooth solution of (1.3).

This paper is devoted to characterizing the class of initial data $u_{0}$ such that the solution of (1.1) may be driven to zero in time $T$ by means of an $L^{2}$ control $v$.

In the case of bounded domains, using Fourier series expansion, the control problem may be reduced to a moment problem (see for instance [5]). However, since we are working on $\mathbb{R}_{+}$, we cannot use Fourier series. Nevertheless, it was observed by M. Escobedo and O. Kavian in [4] that, on suitable similarity variables and at the appropriate scale, solutions of the heat equation on conical domains may be indeed developed in Fourier series on a weighted $L^{2}$ space.

We adapt this idea to our problem. Using similarity variables and weighted Sobolev spaces and developing solutions in Fourier series, we reduce the control problem to a moment problem. We observe that, in the example under consideration (1.1), the spectral density is critical, and this suggests a bad behavior of the moment problem. We prove in fact that, roughly speaking, the moment problem admits an $L^{2}$ solution if and only if the Fourier coefficients grow exponentially as the frequency increases. In particular, this proves that no initial datum $u_{0}$ belonging to any Sobolev space of negative order may be driven to zero in finite time. We complement this negative result by showing that there exist initial data with exponentially growing Fourier coefficients for which null-controllability holds in finite time with $L^{2}$ controls.

In a first reading, this result might seem to be in contradiction with those of B. F. Jones Jr. [14. Indeed, in Corollary 1 of [14 the following is proved: Let $g \in C\left(\mathbb{R}^{n}\right)$ and $T>0$. Then there exists a continuous function $u$ on $\mathbb{R}^{n} \times[0, \infty)$ such that $u$ solves the heat equation in $\mathbb{R}^{n} \times(0, \infty)$, takes the initial datum $g$ at $t=0$ and satisfies the null-control condition $u(x, T) \equiv 0$.

In particular, when $n=1$, by restricting $u$ to the right half-line we see that $v(t)=u(0, t)$, which belongs to $L^{2}(0, T)$, provides a control driving the solution on the half-line to zero in time $T$.

Note however that the solution $u$ constructed in [14] grows very fast at infinity, and therefore it is not the solution one obtains by transposition. In the result of [14], the fast growth rate of solutions as $|x| \rightarrow \infty$ provides a "hidden" control at infinity and allows the null-control condition to be achieved.

The article is organized as follows. In Section 2 we introduce the similarity variables. In Section 3 we define the solutions, derive the corresponding moment 
problem, and state and prove the main results. Finally, in Section 4, we briefly comment on the multi-dimensional case and discuss some other extensions of the results of this paper and open problems. In particular, we describe why the results we prove are in agreement with those of [7], 11] and [24], in which it is shown that, in the case of bounded domains, the control needed to drive the system to rest blows up exponentially as the control time tends to zero.

\section{Similarity variables and Weighted Sobolev Spaces}

In this section we recall some basic facts about the similarity variables and weighted Sobolev spaces for the heat equation. We refer to [4] and [15] for further developments and details.

2.1. The similarity variables. We consider the solutions $u=u(x, t)$ of

$$
\begin{cases}u_{t}(x, t)-u_{x x}(x, t)=0 & \text { for } x \in \Omega, 0<t<T, \\ u(0, t)=v(t) & \text { for } 0<t<T \\ u(x, 0)=u_{0}(x) & \text { for } x \in \Omega\end{cases}
$$

where $\Omega=\mathbb{R}_{+}=(0, \infty)$.

We now introduce the new space-time variables

$$
y=x / \sqrt{t+1}, \quad s=\log (t+1) .
$$

Then, given a solution $u=u(x, t)$ of (2.1), we introduce

$$
w(y, s)=e^{s / 2} u\left(e^{s / 2} y, e^{s}-1\right) .
$$

It follows that $u$ solves (2.1) if and only if $w$ satisfies

$$
\begin{cases}w_{s}(y, s)-w_{y y}(y, s)-\frac{1}{2} y w_{y}(y, s)-\frac{1}{2} w(y, s)=0 & \text { for } y \in \Omega, 0<s<S \\ w(0, s)=\widetilde{v}(s) & \text { for } 0<s<S \\ w(y, 0)=u_{0}(y) & \text { for } y \in \Omega\end{cases}
$$

where

$$
\widetilde{v}(s)=e^{s / 2} v\left(e^{s}-1\right)
$$

and $S=\log (T+1)$.

Obviously, analyzing the null controllability of system (2.1) in time $T$ is equivalent to studying the null controllability of system (2.4) in time $S=\log (T+1)$. Therefore, in the sequel, we shall analyze system (2.4).

The elliptic operator involved in (2.4) may also be written as

$$
L w:=-w_{y y}-\frac{1}{2} y w_{y}=-\frac{1}{K(y)}\left(K(y) w_{y}\right)_{y},
$$

where $K=K(y)$ is the Gaussian weight

$$
K(y)=\exp \left(y^{2} / 4\right)
$$

We first analyze this operator on the whole real axis. 
2.2. Weighted spaces and spectral analysis on the whole real axis. We introduce the weighted $L^{2}$ space

$$
L^{2}(K)=\left\{f: \mathbb{R} \rightarrow \mathbb{R}: \sqrt{K} f \in L^{2}(\mathbb{R})\right\}
$$

endowed with the natural norm

$$
\|f\|_{L^{2}(K)}=\left(\int_{\mathbb{R}}|f(y)|^{2} K(y) d y\right)^{1 / 2} .
$$

Obviously, it is a Hilbert space.

We then define the unbounded operator $L$ on $L^{2}(K)$ by setting

$$
L w=-w_{y y}-\frac{1}{2} y w_{y},
$$

as above, and $D(L)=\left\{w \in L^{2}(K): L w \in L^{2}(K)\right\}$.

Integrating by parts, we can easily see that

$$
\int_{\mathbb{R}}(L w) w K d y=\int_{\mathbb{R}}\left|w_{y}\right|^{2} K d y .
$$

Therefore it is natural to introduce the weighted $H^{1}$ space

$$
H^{1}(K)=\left\{f \in L^{2}(K): f_{y} \in L^{2}(K)\right\}
$$

endowed with the norm

$$
\|f\|_{H^{1}(K)}=\left[\int_{\mathbb{R}}\left(|f|^{2}+\left|f_{y}\right|^{2}\right) K d y\right]^{1 / 2} .
$$

In a similar way, for any $s \in \mathbb{N}$ we may introduce the space

$$
H^{s}(K)=\left\{f \in L^{2}(K): D^{\alpha} f \in L^{2}(K), \alpha=1,2, \ldots, s\right\} .
$$

The following properties were proved in [4] and [15]:

$$
\begin{aligned}
& \int f^{2}|y|^{2} K d y \leq 16 \int\left|f_{y}\right|^{2} K d y, \quad \forall f \in H^{1}(K) ; \\
& \text { the embedding } H^{1}(K) \hookrightarrow L^{2}(K) \text { is compact; } \\
& L: H^{1}(K) \rightarrow\left(H^{1}(K)\right)^{\prime} \text { is an isomorphism; }
\end{aligned}
$$

Moreover, $L$ can be defined as an unbounded operator in $L^{2}(K), L: D(L)=$ $H^{2}(K) \subset L^{2}(K) \rightarrow L^{2}(K)$. In this case $L^{-1}: L^{2}(K) \rightarrow L^{2}(K)$ is self-adjoint and compact. The eigenvalues of $L$ are $\lambda_{j}=\frac{j}{2}, j \geq 1$, and the corresponding eigenfuntions are $\varphi_{j}=D^{(j-1)} \varphi_{1}, j \geq 1$, where $\varphi_{1}$ is the eigenfunction associated to the first eigenvalue $\lambda_{1}$, which is simple, and is explicitly given by

$$
\varphi_{1}(y)=K^{-1}(y)=\exp \left(-y^{2} / 4\right) .
$$

Using this spectral decomposition, the solutions of the heat equation in similarity variables on the whole axis can be easily developed in Fourier series. Namely, if $w$ solves

$$
\left\{\begin{array}{lll}
w_{s}+L w-\frac{1}{2} w=0 & \text { in } & \mathbb{R} \times(0, \infty) \\
w(y, 0)=u_{0}(y) & \text { in } & \mathbb{R}
\end{array}\right.
$$

with

$$
u_{0}(y)=\sum_{j=1}^{\infty} a_{j} \varphi_{j}(y)
$$


then

$$
w(y, s)=\sum_{j=1}^{\infty} e^{-\lambda_{j} s} a_{j} \varphi_{j}(y)
$$

2.3. Spectral analysis on the half-line. This similarity transformation may also be used in $\mathbb{R}_{+}$. The operator $L$ with Dirichlet boundary conditions has basically the same properties as above, except for the fact that the spectrum is not the same (see [15]).

However, the spectrum may easily be computed explicitly. We deduce that $\varphi_{j}$ is an eigenfunction of $L$ in $\Omega=(0, \infty)$ with Dirichlet boundary conditions on the left extreme $y=0$, i.e. such that $\varphi_{j}(0)=0$, if and only if $j$ is even. Consequently, in this case the eigenfunctions are

$$
\widehat{\phi}_{m}(y)=\varphi_{2 m}(y), \quad m \geq 1,
$$

and the corresponding eigenvalues are

$$
\omega_{m}=\lambda_{2 m}=\frac{2 m}{2}=m, \quad m \geq 1 .
$$

Here and in the sequel, by $L^{2}\left(\mathbb{R}_{+} ; K\right)$ we denote the weighted $L^{2}$ space

$$
L^{2}\left(\mathbb{R}_{+} ; K\right)=\left\{f: \mathbb{R}_{+} \rightarrow \mathbb{R}: \sqrt{K} f \in L^{2}\left(\mathbb{R}_{+}\right)\right\}
$$

endowed with the canonical norm. We will also use the weighted Sobolev spaces

$$
H^{1}\left(\mathbb{R}_{+} ; K\right)=\left\{f \in L^{2}\left(\mathbb{R}_{+} ; K\right): f_{y} \in L^{2}\left(\mathbb{R}_{+} ; K\right)\right\}
$$

and

$$
H_{0}^{1}\left(\mathbb{R}_{+} ; K\right)=\left\{f \in H^{1}\left(\mathbb{R}_{+} ; K\right): f(0)=0\right\},
$$

endowed with the canonical norms. Finally, by $H^{-1}\left(\mathbb{R}_{+} ; K\right)$ we denote the dual of $H_{0}^{1}\left(\mathbb{R}_{+} ; K\right)$.

Let us define $\phi_{m}(y)=C_{m} \phi_{m}(y)$ such that $\left\|\phi_{m}\right\|_{L^{2}\left(\mathbb{R}_{+} ; K\right)}=1$. We shall give an estimate for the constant $C_{m}$ in section 3.3.

Observe that $\left(\phi_{m}\right)_{m \geq 1}$ forms an orthonormal basis in $L^{2}\left(\mathbb{R}_{+} ; K\right)$.

\section{The Control Problem}

In this section we analyze the control problem in the half-line:

$$
\begin{cases}u_{t}-u_{x x}=0, & x>0, \quad t>0, \\ u(0, t)=v(t), & t>0 \\ u(x, 0)=u_{0}(x), & x>0\end{cases}
$$

Here, $u=u(x, t)$ is the state and $v=v(t)$ is the control.

Given $T>0$, we are interested in the structure of the space of initial data that may be driven to zero in time $T$ by means of a control $v \in L^{2}(0, T)$. In other words, we want to describe the space of data $u_{0}$ for which there exists $v \in L^{2}(0, T)$ such that the solution of (3.1) satisfies

$$
u(x, T)=0, \quad \forall x>0 .
$$


We define $w$ by means of the similarity transformation (2.3). Then, $u$ solves (3.1) and satisfies (3.2) if and only if $w$ solves

$$
\begin{cases}w_{s}-w_{y y}-\frac{y w_{y}}{2}-\frac{1}{2} w=0, & y>0,0<s<S, \\ w(0, s)=\widetilde{v}(s), & 0<s<S, \\ w(y, 0)=u_{0}(y), & y>0,\end{cases}
$$

and satisfies

$$
w(y, S)=0, \quad \forall y>0,
$$

with

$$
S=\log (T+1)
$$

and $\widetilde{v}$ as in (2.5).

Obviously, $v \in L^{2}(0, T)$ if and only if $\widetilde{v} \in L^{2}(0, S)$. Therefore, the problem is reduced to analyzing the structure of the space of initial data $u_{0}$ for which there exists $\widetilde{v} \in L^{2}(0, S)$ such that the solution of (3.3) satisfies (3.4).

The following section provides a functional setting guaranteeing that the system (3.3) is well posed. Our control results refer to the solutions of $w$ defined by transposition. Indeed, the definition of the solution is used in an essential way when we reduce the control problem to a moment problem.

3.1. Solutions by transposition. As we have mentioned in the introduction, unless some growth conditions are imposed at infinity, equation (3.3) has multiple smooth solutions (see 13, pp. 171-176). Moreover, as Jones [14] has shown, there are solutions of the heat equation that vanish identically on $\mathbb{R}_{+}$at $t=T$. This provides examples of smooth, null-controllable initial data. Nevertheless, the corresponding solutions grow very rapidly at infinity and do not belong to the class of solutions we shall be dealing with.

Hence it is necessary to give a precise definition of solution. The frame in which we shall work is that of 'solutions by transposition' (see [17]).

Moreover, some of the initial data taken under consideration will be very irregular - belonging to some dual space - and a notion of ultraweak solution has to be introduced. The main point here is that, by using spectral decomposition, a solution as in (2.13) can be defined even for exponentially growing Fourier coefficients.

Let $\delta>0$ be a positive real number, and define the space

$$
\mathcal{X}_{\delta}=\left\{u=\sum_{m \geq 1} a_{m} \phi_{m}: \sum_{m \geq 1}\left|a_{m}\right|^{2} e^{2 \delta \omega_{m}}<\infty\right\} .
$$

Here $\left(\phi_{m}\right)_{m \geq 1}$ is the orthonormal basis in $L^{2}\left(\mathbb{R}_{+} ; K\right)$ defined in section 2.3.

Obviously, $\mathcal{X}_{\delta}$ is a Hilbert space with the inner product defined by

$$
(u, v)=\sum_{m \geq 1} a_{m} b_{m} e^{2 \delta \omega_{m}} \quad \text { if } u=\sum_{m \geq 1} a_{m} \phi_{m} \text { and } v=\sum_{m \geq 1} b_{m} \phi_{m},
$$

and $\mathcal{X}_{\delta} \subset H^{s}\left(\mathbb{R}_{+} ; K\right) \subset L^{2}\left(\mathbb{R}_{+} ; K\right)=\mathcal{X}_{0}$ for all $\delta>0$ and $s>0$.

Note that $\mathcal{X}_{\delta}$ can be identified with the space of sequences

$$
\left\{\left(a_{m}\right)_{m \geq 1} \in \ell^{2}: \sum_{m \geq 1}\left|a_{m}\right|^{2} e^{2 \delta \omega_{m}}<\infty\right\} .
$$


The dual of $\mathcal{X}_{\delta}$ will be denoted by $\mathcal{X}_{\delta}^{*}$, and the corresponding dual product by $\langle\cdot, \cdot\rangle_{\delta}$.

Observe that each $u \in \mathcal{X}_{\delta}^{*}$ can be written in the following way:

$$
u=\sum_{m \geq 1} a_{m} \phi_{m}^{*}
$$

where $\phi_{m}^{*} \in \mathcal{X}_{\delta}^{*}$ is the dual basis defined by $\left\langle\phi_{m}^{*}, w\right\rangle_{\delta}=\int_{0}^{\infty} w(y) \phi_{m}(y) K(y) d y$ for all $w \in \mathcal{X}_{\delta}$, and $a_{m}=\left\langle u, \phi_{m}\right\rangle_{\delta}$.

Hence, $\mathcal{X}_{\delta}^{*}$ can be also identified with the space of sequences

$$
\left\{\left(a_{m}\right)_{m \geq 1}: \sum_{m \geq 1}\left|a_{m}\right|^{2} e^{-2 \delta \omega_{m}}<\infty\right\} .
$$

We shall define by transposition the solutions of (3.3) with initial data in $\mathcal{X}_{\delta}^{*}$ and non-homogeneous boundary term $\widetilde{v} \in L^{2}(0, S)$.

Let us first consider the non-homogeneous adjoint equation

$$
\begin{cases}\xi_{s}+\xi_{y y}+y \frac{\xi_{y}}{2}+\frac{1}{2} \xi=h, & y>0, \quad 0<s<S, \\ \xi(0, s)=0, & 0<s<S, \\ \xi(y, S)=\xi_{0}(y), & y>0 .\end{cases}
$$

Multiplying in (3.3) by $\xi K$ and integrating by parts, we deduce that

$$
\int_{0}^{S} \int_{0}^{\infty} h w K d y d s=\left.\int_{0}^{\infty} \xi w K d y\right|_{0} ^{S}+\int_{0}^{S} \xi_{y}(0, s) \widetilde{v}(s) d s
$$

i.e.

$$
\begin{aligned}
\int_{0}^{S} & \int_{0}^{\infty} h w K d y \\
& =\int_{0}^{\infty} \xi_{0}(y) w(y, S) K d y-\int_{0}^{\infty} \xi(y, 0) u_{0}(y) K d y+\int_{0}^{S} \xi_{y}(0, s) \widetilde{v}(s) d s .
\end{aligned}
$$

Of course, the integrations by parts leading to (3.7) are justified provided both $\xi$ and $w$ are smooth enough and do not grow too fast as $y \rightarrow \infty$. Therefore, this is by now a formal computation. We use (3.7) to define the solution of (3.3) in the sense of transposition.

More precisely, given $\widetilde{v} \in L^{2}(0, S)$ and $u_{0} \in \mathcal{X}_{\delta}^{*}$, we say that $w \in C\left([0, S] ; \mathcal{X}_{\delta}^{*}\right)$ is a solution of (3.3) in the sense of transposition if the following equality holds:

$$
\int_{0}^{S}\langle w, h\rangle_{\delta} d t=\left\langle w(\cdot, S), \xi_{0}\right\rangle_{\delta}-\left\langle u_{0}, \xi(\cdot, 0)\right\rangle_{\delta}+\int_{0}^{S} \xi_{y}(0, s) \widetilde{v}(s) d s
$$

for any $\xi_{0} \in \mathcal{X}_{\delta}, h \in L^{1}\left([0, S] ; \mathcal{X}_{\delta}\right)$, and $\xi \in C\left([0, S], \mathcal{X}_{\delta}\right)$ the corresponding solution of (3.6).

The following holds:

Proposition 3.1. For any $u_{0} \in \mathcal{X}_{\delta}^{*}$ and $\widetilde{v} \in L^{2}(0, S)$ there exists a unique solution $w \in C\left([0, S] ; \mathcal{X}_{\delta}^{*}\right)$ of $(3.3)$ in the sense of transposition.

Proof. Let us first remark that the uniqueness of a solution of (3.8) follows immediately by taking $\xi_{0}=0$ and $h(y, s)=h_{1}(s) h_{2}(y)$ with $h_{1} \in L^{1}(0, S)$ and $h_{2} \in \mathcal{X}_{\delta}$.

For the existence, due to the linearity of the system (3.3), it is sufficient to consider the following two cases: i) $u_{0} \equiv 0$ and ii) $\widetilde{v}=0$. 
Case i): We prove that (3.8) with $u_{0}=0$ has a solution

$$
w^{1} \in C\left([0, S] ; H^{-1}\left(\mathbb{R}_{+} ; K\right)\right) .
$$

Since $H^{-1}\left(\mathbb{R}_{+} ; K\right) \subset \mathcal{X}_{\delta}^{*}$, it will follow that $w^{1} \in C\left([0, S] ; \mathcal{X}_{\delta}^{*}\right)$.

Classical energy estimates for the solutions of (3.6) show that there exists $C>0$ such that

$$
\begin{aligned}
& \|\xi\|_{L^{\infty}\left(0, S ; H_{0}^{1}\left(\mathbb{R}_{+} ; K\right)\right)}+\|\xi\|_{L^{2}\left(0, S ; H^{2} \cap H_{0}^{1}\left(\mathbb{R}_{+} ; K\right)\right)} \\
& \quad \leq C\left[\|h\|_{L^{1}\left(0, S ; H_{0}^{1}\left(\mathbb{R}_{+} ; K\right)\right)}+\left\|\xi_{0}\right\|_{H_{0}^{1}\left(\mathbb{R}_{+} ; K\right)}\right], \\
& \forall h \in L^{1}\left(0, S ; H_{0}^{1}\left(\mathbb{R}_{+} ; K\right)\right), \xi_{0} \in H_{0}^{1}\left(\mathbb{R}_{+} ; K\right) .
\end{aligned}
$$

By duality, we deduce the existence of $w^{1} \in L^{\infty}\left(0, S ; H^{-1}\left(\mathbb{R}_{+} ; K\right)\right)$ and $\zeta^{1} \in$ $H^{-1}\left(\mathbb{R}_{+} ; K\right)$ such that

$$
\begin{aligned}
& \int_{0}^{S}\left\langle w^{1}, h\right\rangle d s-\left\langle\zeta^{1}, \xi_{0}\right\rangle=\int_{0}^{S} \xi_{y}(0, s) \widetilde{v}(s) d s \\
& \forall h \in L^{1}\left(0, S ; H_{0}^{1}\left(\mathbb{R}_{+} ; K\right)\right), \xi_{0} \in H_{0}^{1}\left(\mathbb{R}_{+} ; K\right),
\end{aligned}
$$

where $\langle\cdot, \cdot\rangle$ is the duality product between $H^{1}\left(\mathbb{R}_{+} ; K\right)$ and $H^{-1}\left(\mathbb{R}_{+} ; K\right)$.

Moreover, the following estimate holds:

$$
\left\|w^{1}\right\|_{L^{\infty}\left(0, S ; H^{-1}\left(\mathbb{R}_{+} ; K\right)\right)}+\left\|\zeta^{1}\right\|_{H^{-1}\left(\mathbb{R}_{+} ; K\right)} \leq C\|\widetilde{v}\|_{L^{2}(0, S)}, \quad \forall \widetilde{v} \in L^{2}(0, S) .
$$

This is because, in view of (3.9), one also has

$$
\begin{aligned}
& \left|\int_{0}^{S} \xi_{y}(0, S) \widetilde{v}(s) d s\right| \leq\left\|\xi_{y}(0, S)\right\|_{L^{2}(0, S)}\|\widetilde{v}\|_{L^{2}(0, S)} \\
& \quad \leq C\|\widetilde{v}\|_{L^{2}(0, S)}\left[\|h\|_{L^{1}\left(0, S ; H_{0}^{1}\left(\mathbb{R}_{+} ; K\right)\right)}+\left\|\xi_{0}\right\|_{H_{0}^{1}\left(\mathbb{R}_{+} ; K\right)}\right] .
\end{aligned}
$$

In view of (3.11) and by a classical density argument it is easy to see that, in fact, $w^{1} \in C\left([0, S] ; H^{-1}\left(\mathbb{R}_{+} ; K\right)\right)$.

To conclude, it is sufficient to prove that

$$
w^{1}(S)=\zeta^{1} \in H^{-1}\left(\mathbb{R}_{+}, K\right) \subset \mathcal{X}_{\delta}^{*} .
$$

This can be done easily by taking solutions of (3.6) corresponding to data

$$
\begin{cases}\xi_{0}=\phi_{m}, & m \geq 1, \\ h_{\delta}(y, s)=a_{\delta}(s) \phi_{m}, & m \geq 1, \\ a_{\delta}(s)= \begin{cases}\frac{1}{\delta}, & S-\delta \leq s \leq S, \\ 0, & 0 \leq s \leq S-\delta,\end{cases} \end{cases}
$$

$\phi_{m}$ being the eigenfunctions of $L$ constituting an orthonormal basis of $L^{2}\left(\mathbb{R}^{+} ; K\right)$ described in section 2.3.

Indeed, applying (3.26) with this choice of $\xi_{0}$ and $h=h_{\delta}$ (and, therefore, the corresponding solution of (3.6) ) , one deduces that

$$
\lim _{\delta \rightarrow 0} \frac{1}{\delta} \int_{S-\delta}^{S}\left\langle w^{1}(s), \phi_{m}\right\rangle d s=\left\langle\zeta^{1}, \phi_{m}\right\rangle, \quad \forall m \geq 1
$$

which, of course, implies (3.12).

Case ii): We prove that (3.8) with $\widetilde{v}=0$ has a solution $w^{2} \in C\left([0, S] ; \mathcal{X}_{\delta}^{*}\right)$. 
Equation (3.6) is well-posed for $\xi_{0} \in \mathcal{X}_{\delta}$ and $h \in L^{1}\left(0, T ; \mathcal{X}_{\delta}\right)$. The corresponding solution, $\xi$, belongs to $C\left([0, T], \mathcal{X}_{\delta}\right)$ and is continuous with respect to $\xi_{0}$ and $h$ :

$$
\|\xi\|_{L^{\infty}\left(0, S ; \mathcal{X}_{\delta}\right)} \leq C\left[\|h\|_{L^{1}\left(0, S ; \mathcal{X}_{\delta}\right)}+\left\|\xi_{0}\right\|_{\mathcal{X}_{\delta}}\right], \quad \forall h \in L^{1}\left(0, S ; \mathcal{X}_{\delta}\right), \xi_{0} \in \mathcal{X}_{\delta} .
$$

It follows by duality that there exist $w^{2} \in L^{\infty}\left(0, S ; \mathcal{X}_{\delta}^{*}\right)$ and $\zeta^{2} \in \mathcal{X}_{\delta}^{*}$ such that

$$
\begin{gathered}
\int_{0}^{S}\left\langle w^{2}, h\right\rangle_{\delta} d s-\left\langle\zeta^{2}, \xi_{0}\right\rangle_{\delta}+\left\langle u_{0}, \xi(0)\right\rangle_{\delta}=0 \\
\forall h \in L^{1}\left(0, S ; \mathcal{X}_{\delta}\right), \xi_{0} \in \mathcal{X}_{\delta}
\end{gathered}
$$

Moreover, the following estimate holds:

$$
\left\|w^{2}\right\|_{L^{\infty}\left(0, S ; \mathcal{X}_{\delta}^{*}\right)}+\left\|\zeta^{2}\right\|_{\mathcal{X}_{\delta}^{*}} \leq C\left\|u_{0}\right\|_{\mathcal{X}_{\delta}^{*}}, \quad \forall u_{0} \in \mathcal{X}_{\delta}^{*} .
$$

This is because one also has

$$
\begin{aligned}
& \left|\int_{0}^{S} \xi_{y}(0, S) \widetilde{v}(s) d s\right| \leq\left\|\xi_{y}(0, S)\right\|_{L^{2}(0, S)}\|\widetilde{v}\|_{L^{2}(0, S)} \\
& \quad \leq C\|\widetilde{v}\|_{L^{2}(0, S)}\left[\|h\|_{L^{1}\left(0, S ; H_{0}^{1}\left(\mathbb{R}_{+} ; K\right)\right)}+\left\|\xi_{0}\right\|_{H_{0}^{1}\left(\mathbb{R}_{+} ; K\right)}\right] \\
& \quad \leq C\|\widetilde{v}\|_{L^{2}(0, S)}\left[\|h\|_{L^{1}\left(0, S ; \mathcal{X}_{\delta}\right)}+\left\|\xi_{0}\right\|_{\mathcal{X}_{\delta}}\right] .
\end{aligned}
$$

In view of (3.16) and by a classical density argument it is easy to see that, in fact, $w^{2} \in C\left([0, S] ; \mathcal{X}_{\delta}^{*}\right)$.

As in case i), one also proves that $w^{2}(S)=\zeta^{2}$.

The proof of Proposition 3.1 is finished now by taking $w=w^{1}+w^{2}$.

Remark 3.1. Observe that the solution $w^{2}$ of (3.3) with $\widetilde{v}=0$ can also be obtained by extending the semigroup corresponding to (3.3) to $\mathcal{X}_{\delta}^{*}$.

In fact, if $u_{0}(y)=\sum_{j=1}^{\infty} a_{j} \phi_{j}(y)$ is the initial datum of (3.3), then the corresponding solution will be $w(y, s)=\sum_{j=1}^{\infty} e^{-\omega_{j} s} a_{j} \phi_{j}(y)$, where all the series converge in $\mathcal{X}_{\delta}^{*}$.

Remark 3.2. One can also define the solutions of (3.1) directly by transposition (in the original space-time variables $(x, t)$ ). It turns out that for any $u_{0} \in L^{2}\left(\mathbb{R}_{+}\right)$and $v \in L^{2}(0, T)$ there exists a unique solution of (3.1) in the sense of transposition such that $u \in C\left([0, T] ; H^{-1}\left(\mathbb{R}_{+}\right)\right)$.

However, when $u_{0} \in L^{2}\left(\mathbb{R}_{+} ; K\right)$ one can also obtain the solution of (3.1) from the solution of (3.3) given by Proposition 3.1 by undoing the change of variables of section 2.1 Indeed, $u$ may be written as

$$
u(x, t)=(t+1)^{-1 / 2} w\left(\frac{x}{\sqrt{t+1}}, \log (t+1)\right) .
$$

Obviously, in this case, the two solutions of (3.1) (the one obtained directly by transposition and the one obtained by the change of variables (3.17) from the solution $w$ of (3.3) in the sense of transposition) coincide.

3.2. Main results on the moment and control problems. We first reduce the control problem to a moment problem. 
Proposition 3.2. The initial datum $u_{0}$ of system (3.3) is null-controllable in time $S$ if and only if there exists $\widetilde{v} \in L^{2}(0, S)$ such that

$$
\int_{0}^{S} \widetilde{v}(s) e^{(m-1 / 2) s} d s=\frac{a_{m}}{\phi_{m}^{\prime}(0)}, \quad \forall m \geq 1,
$$

where $a_{m}=\left\langle u_{0}, \phi_{m}\right\rangle_{\delta}$.

Remark 3.3. Note that, when $u_{0} \in L^{2}\left(\mathbb{R}_{+} ; K\right)$, we have

$$
\left\langle u_{0}, \phi_{m}\right\rangle_{\delta}=\int_{0}^{\infty} u_{0}(y) \phi_{m}(y) K(y) d y
$$

for all $m \geq 1$.

Proof of Proposition 3.2. Let us first suppose that $u_{0}$ is null-controllable. From (3.8) it follows that

$$
\int_{0}^{S}\langle w, h\rangle_{\delta} d t+\left\langle u_{0}, \xi(\cdot, 0)\right\rangle_{\delta}=\int_{0}^{S} \xi_{y}(0, s) \widetilde{v}(s) d s
$$

for any solution of (3.6) such that $\xi_{0} \in \mathcal{X}_{\delta}$ and $h \in L^{1}\left([0, S] ; \mathcal{X}_{\delta}\right)$.

By taking $h=0$ and $\xi_{0}=\phi_{m}, m \geq 1$, we see that the corresponding solution of (3.6) is $\xi(y, s)=e^{(-m+1 / 2)(S-s)} \phi_{m}(y)$. With this choice, from (3.19), we obtain

$$
e^{(-m+1 / 2) S} a_{m}=\int_{0}^{S} e^{(-m+1 / 2)(S-s)} \phi_{m}^{\prime}(0) \widetilde{v}(s), \quad m \geq 1,
$$

which is equivalent to (3.18).

On the other hand, suppose that (3.18) holds. By taking again $h=0$ and $\xi_{0}=\phi_{m}, m \geq 1$, we obtain from (3.8) that

$$
\left\langle w(\cdot, S), \phi_{m}\right\rangle_{\delta}=0, \quad m \geq 1 .
$$

Hence, $w(\cdot, S)=0$, and therefore $u_{0}$ is controllable.

According to Proposition 3.2 the control problem is reduced to analyzing the sequences $\left\{a_{m}\right\}_{m \geq 1}$ such that (3.18) holds for some $\widetilde{v} \in L^{2}(0, S)$.

We set

$$
\alpha_{m}=a_{m} / \phi_{m}^{\prime}(0)
$$

and

$$
f(s)=\widetilde{v}(s) e^{S / 2} .
$$

Obviously, $\widetilde{v} \in L^{2}(0, S)$ if and only if $f \in L^{2}(0, S)$. On the other hand, (3.18) is equivalent to

$$
\int_{0}^{S} f(s) e^{m s} d s=\alpha_{m}, \quad \forall m \geq 1 .
$$

This is the moment problem we shall address.

We now state the main result of this paper on the moment problem (3.22).

Theorem 3.1. a) Assume that the coefficients $\left\{\alpha_{m}\right\}$ are such that there exist $\delta>0$ and $C_{\delta}>0$ such that

$$
\left|\alpha_{m}\right| \leq C_{\delta} e^{\delta m}, \quad \forall m \geq 1 .
$$

If (3.22) holds for some $S>0$ and $f \in L^{2}(0, S)$, then, necessarily, $\operatorname{supp}(f) \subseteq[0, \delta]$. 
b) Assume that the coefficients $\left\{\alpha_{m}\right\}$ are such that for any $\delta>0$ there exists $C_{\delta}>0$ such that

$$
\left|\alpha_{m}\right| \leq C_{\delta} e^{\delta m}, \quad \forall m \geq 1
$$

If 3.22) holds for some $S>0$ and $f \in L^{2}(0, S)$, then, necessarily, $\alpha_{m}=0$ for all $m \geq 1$.

c) For any $\delta>0$ and $S>\delta$ there exist sequences $\left\{\alpha_{m}\right\}$ such that

$$
C_{1} e^{\delta m} / m \leq\left|\alpha_{m}\right| \leq C_{2} e^{\delta m} / m, \quad \forall m \geq 1,
$$

for suitable positive constants $C_{1}, C_{2}>0$, and the moment problem (3.22) has a solution $f \in L^{2}(0, S)$ with $\operatorname{supp}(f) \subseteq[0, \delta]$.

Remark 3.4. Statement b) of Theorem 3.1 is a direct consequence of statement a), since the latter implies $\operatorname{supp}(f) \subseteq[0, \delta]$ for all $\delta>0$.

Roughly speaking, b) proves that the coefficients $\left\{\alpha_{m}\right\}$ need to grow exponentially to guarantee the existence of an $L^{2}(0, S)$ solution of the moment problem (3.22). More precisely, b) shows that a necessary condition for the nullcontrollability is

$$
\lim \sup \left\{\left[\log \left|\alpha_{m}\right|\right] / m\right\}>0 .
$$

Statement c) proves the optimality of a) and b).

In order to prove statement c) from Theorem 3.1 we need to rewrite the moment problem in an equivalent way:

Proposition 3.3. The following assertions are equivalent:

(a) There exists $f \in L^{2}(0, S)$ such that (3.22) holds.

(b) There exists an entire function $F$ of exponential type $\leq S / 2$ with

$$
\int_{-\infty}^{\infty}|F(i y)|^{2} d y<\infty
$$

and such that

$$
F(m)=\alpha_{m} e^{-m S / 2}, \quad \forall m \geq 1 .
$$

Remark 3.5. As the proof of this proposition shows (see section 3.3 below), the function $F$ in (b) is uniformly bounded along the imaginary axis.

Remark 3.6. If (3.25) from Theorem 3.1 holds, the corresponding minimal control time is $S=\delta$. Indeed, let us suppose that the moment problem (3.22) has a solution $f \in L^{2}(0, S)$ with $S<\delta$. From Proposition 3.3 we obtain that there exists an entire function $F$ of exponential type $\leq S / 2$, such that $\alpha_{m}=F(m) e^{\frac{m S}{2}}$. Hence

$$
C_{1} e^{\delta m} / m \leq\left|\alpha_{m}\right|=\left|F(m) e^{\frac{m S}{2}}\right| \leq A e^{m(S+\varepsilon)}
$$

for all $\varepsilon>0$, which is impossible if $S<\delta$.

Theorem 3.1 and Proposition 3.3 will be proved in section 3.3.

According to Theorem 3.1 and Proposition 3.3 in order to characterize the nullcontrollable initial data it is necessary and sufficient to characterize the sequences $\{F(m)\}_{m \geq 1}$ that may be obtained by means of entire functions $F$ of exponential type $\leq S / 2$ satisfying (3.26) and (3.27). We shall use this result to prove that there exist null-controllable initial data.

As an immediate consequence of Theorem 3.1 we obtain the following result on the control problem: 
Theorem 3.2. There is no non-trivial initial datum $u_{0}$ which is null-controllable in finite time and with Fourier coefficients $\left\{a_{m}\right\}$ satisfying

$$
\left|a_{m}\right| \leq C_{\delta} e^{\delta m}, \quad \forall m \geq 1,
$$

for all $\delta>0$

Moreover, for any $\delta>0$ and $S>\delta$ there exist non-trivial initial data $u_{0}$ that are null-controllable in time $S$ and such that

$$
C_{1} \frac{e^{\delta m}}{m^{3 / 4}} \leq\left|a_{m}\right| \leq C_{2} \frac{e^{\delta m}}{m^{3 / 4}}, \quad \forall m \geq 1,
$$

for suitable positive constants $C_{1}, C_{2}>0$. In this case a control $f \in L^{2}(0, S)$ with $\operatorname{supp}(f) \subseteq[0, \delta]$ can be found.

Remark 3.7. Note that, if the Fourier coefficients $\left\{a_{m}\right\}_{m>1}$ of the initial datum $u_{0}$ satisfy (3.29), then the the corresponding solution of (3.3) is well-defined and belongs to $C\left([0, S], \mathcal{X}_{\delta^{\prime}}^{*}\right)$ for all $\delta^{\prime}<\delta$ (see section 3.1).

The proof of Theorem 3.2 will be given in section 3.3

Theorem 3.2 indicates, roughly speaking, that null-controllable initial data have exponentially growing Fourier coefficients. Actually, the Fourier coefficients need to be exponentially large for any $0 \leq s<S$ along the controlled trajectory.

Observe that $u_{0} \in H^{-\alpha}\left(\mathbb{R}_{+} ; K\right)$ with $\alpha>0$ if $\sum_{m>1}\left|a_{m}\right|^{2} m^{-\alpha}<\infty$. Consequently the null-controllable initial data that Theorem 3.2 provides satisfying (3.18) do not belong to any Sobolev space of negative order $H^{-\alpha}\left(\mathbb{R}_{+} ; K\right)$.

On the other hand, if $u_{0} \in H^{-\alpha}\left(\mathbb{R}_{+} ; K\right)$ for some $\alpha>0$, we have

$$
\left|a_{m}\right|=\left|\left\langle u_{0}, \phi_{m}\right\rangle\right| \leq\left\|u_{0}\right\|_{H^{-\alpha}\left(\mathbb{R}_{+} ; K\right)}\left\|\phi_{m}\right\|_{H^{\alpha}\left(\mathbb{R}_{+} ; K\right)} .
$$

Taking into account that $\left\|\phi_{m}\right\|_{H^{\alpha}\left(\mathbb{R}_{+} ; K\right)}$ grows polynomialy as $m \rightarrow \infty$, we deduce that (3.28) holds, and therefore $u_{0}$ is not null-controllable, except when $u_{0} \equiv 0$.

Theorem 3.2 refers to the null-controllability of systems (3.3) in the similarity variables. However, due to the equivalence of the null-controllability of systems (3.1) and (3.3), the following holds:

Corollary 3.1. There is no non-trivial initial datum $u_{0}$ which is null-controllable in finite time for system (3.1) by means of $L^{2}$ boundary controls and such that

$$
\left|a_{m}\right| \leq C_{\delta} e^{\delta m}, \quad \forall m \geq 1,
$$

for all $\delta>0$.

Moreover, for any $\delta>0$ and $T>e^{\delta}-1$ there exist non-trivial initial data $u_{0}$ for system (3.1) that are null-controllable in time $T$ with $L^{2}$ controls supported in $[0, \delta]$ and such that its Fourier coefficients $\left\{a_{m}\right\}$ satisfy (3.29).

Remark 3.8. According to Corollary[3.1 we deduce, in particular, that the following initial data are not null-controllable in any time $T$ for system (3.1):

- $u_{0}(x)=x^{k} \exp \left(-x^{2} / 2\right), \forall k \geq 0$;

- $u_{0} \in \mathcal{D}\left(\mathbb{R}_{+}\right)$.

As we mentioned in the introduction, this result is in contrast with the existing ones for bounded domains that guarantee that any initial datum in any Sobolev space of negative order is null-controllable in an arbitrarily short time.

The examples we have mentioned above show that the lack of null-controllability on the half-line is not due to the lack of regularity or to the lack of decay at infinity 
of the initial data. In fact, there is no initial datum $u_{0} \in \mathcal{D}\left(\mathbb{R}_{+}\right)$, except for the trivial one, that is null-controllable in any time!

The proof of Theorem 3.2 we present below provides an explanation of this unusual fact. Roughly speaking, an $L^{2}$ control produces an exponential effect on the Fourier coefficients of the controlled trajectory for any time, as the frequency increases. Thus, null-controllability may only be achieved when the Fourier coefficients of the initial datum are already exponentially large.

Note that when (3.25) holds, $S=\delta$ is the minimal control time for these initial data (see also Remark 3.6).

As we mentioned in the introduction, this result might seem to be in contradiction with those in [14] that guarantee that for any initial datum $u_{0} \in C\left(\mathbb{R}^{n}\right)$ and $T>0$ there exists a continuous solution $u$ of the heat equation in $\mathbb{R}^{n} \times[0, \infty)$ such that $u(x, T) \equiv 0$. However, this solution $u$ grows very fast at infinity, and therefore it is not the solution one obtains by transposition, which is the frame in which we are working.

3.3. Proofs of the main results. This section is devoted to proving Propositions 3.3, Theorem 3.1 and Theorem 3.2 .

Proof of Proposition [3.3. First of all we observe that

$$
\begin{aligned}
& \int_{0}^{S} f(s) e^{m s} d s=\int_{-S / 2}^{S / 2} f(s+S / 2) e^{m(s+S / 2)} d s \\
& =e^{m S / 2} \int_{-S / 2}^{S / 2} f(s+S / 2) e^{m s} d s=e^{m S / 2} \int_{-S / 2}^{S / 2} g(s) e^{m s} d s
\end{aligned}
$$

with $g(s)=f(s+S / 2)$. Hence, statement (a) of Proposition 3.3 is equivalent to

$$
\text { (a') } \exists g \in L^{2}(-S / 2, S / 2) \text { such that } \int_{-S / 2}^{S / 2} g(s) e^{m s} d s=e^{-m S / 2} \alpha_{m}, \forall m \geq 1 .
$$

We now prove that $\left(\mathrm{a}^{\prime}\right)$ and $(\mathrm{b})$ are equivalent.

$\left(\mathrm{a}^{\prime}\right) \Rightarrow(\mathrm{b})$. Let $G$ be the Fourier transform of $g$ or, more precisely, of $g(s) 1_{(-S / 2, S / 2)}$, i.e. $G(z)=\int_{-S / 2}^{S / 2} g(s) e^{-i z s} d s$, and let $F(z)=G(i z)$. According to the Paley-Wiener Theorem (see for instance [2]), we know that $G: \mathbf{C} \rightarrow \mathbf{C}$ is an entire function of exponential type $\leq S / 2$ and such that $\int_{-\infty}^{\infty}|G(x)|^{2} d x<\infty$. Consequently, $F$ is also an entire function of exponential type $\leq S / 2$ such that $\int_{-\infty}^{\infty}|F(i x)|^{2} d x<\infty$.

Moreover, in view of (3.31),

$$
F(m)=G(i m)=\int_{-S / 2}^{S / 2} g(s) e^{m s} d s=e^{-m S / 2} \alpha_{m} .
$$

This shows that (b) holds.

(b) $\Rightarrow\left(\mathrm{a}^{\prime}\right)$. Let $F$ be an entire function of exponential type $\leq S / 2$, with $\int_{-\infty}^{\infty}|F(i x)|^{2} d x<\infty$ and such that (3.27) holds. We then set $G(z)=F(-i z)$, which is also an entire function of exponential type $\leq S / 2$ with $\int_{-\infty}^{\infty}|G(x)|^{2} d x<$ $\infty$. 
From the Paley-Wiener Theorem we deduce that there exist $g \in L^{2}(-S / 2, S / 2)$ such that

$$
G(z)=\int_{-S / 2}^{S / 2} g(s) e^{-i z s} d s .
$$

Then, the function $f(s)=g(s+S / 2)$ is such that

$$
\int_{0}^{S} f(s) e^{m s} d s=\int_{-S / 2}^{S / 2} g(s) e^{m(s+S / 2)} d s=e^{m S / 2} G(i m)=e^{m S / 2} F(m)=\alpha_{m},
$$

by (3.27). This completes the proof of Proposition 3.3

Proof of Theorem 3.1. a) For the proof of the first statement a method due to RyllNardzevski, used by Yosida [27] (pp. 166-167) in a similar context, can be applied. First of all let us give the following lemma:

Lemma 3.1. If $g \in L^{2}(0, T)$ and $0 \leq t \leq T$, then

$$
\lim _{x \rightarrow \infty} \sum_{k=1}^{\infty} \frac{(-1)^{k-1}}{k !} \int_{0}^{T} e^{k x(t-u)} g(u) d u=\int_{0}^{t} g(u) d u .
$$

Proof. The case $g \in C[0, T]$ is proved in Yosida [27] (pp. 166-167). For the sake of completeness we recall that identity (3.32) holds by the Taylor expansion of the function $1-\exp \left(-e^{x(t-u)}\right)$ and the dominated convergence theorem. Exactly the same proof also holds if $g \in L^{2}(0, T)$.

The following lemma is also inspired by Yosida [27.

Lemma 3.2. If $g \in L^{2}(0, T)$ is such that there exist two positive constants $\delta>0$ and $C_{\delta}>0$ such that

$$
\left|\int_{0}^{T} g(u) e^{m u} d u\right| \leq C_{\delta} e^{m \delta}, \quad \forall m \geq 1,
$$

then $\operatorname{supp}(g) \subseteq[0, \delta]$.

Proof. From the previous lemma we have

$$
\lim _{m \rightarrow \infty} \sum_{k=1}^{\infty} \frac{(-1)^{k-1}}{k !} \int_{0}^{T} e^{k m(t-u)} g(T-u) d u=\int_{0}^{t} g(T-u) d u .
$$

But

$$
\begin{gathered}
\left|\sum_{k=1}^{\infty} \frac{(-1)^{k-1}}{k !} \int_{0}^{T} e^{k m(t-u)} g(T-u) d u\right| \\
\leq \sum_{k=1}^{\infty} \frac{1}{k !} e^{k m(t-T)}\left|\int_{0}^{T} e^{k m \tau} g(\tau) d \tau\right| \\
\leq \sum_{k=1}^{\infty} \frac{1}{k !} e^{k m(t-T)} C_{\delta} e^{k m \delta}=C_{\delta}\left(\exp \left(e^{m(t-T+\delta)}\right)-1\right) .
\end{gathered}
$$

Since the last expression tends to zero as $m$ tends to infinity for $t \leq T-\delta$, it follows that $\int_{0}^{t} g(T-u) d u=0,0 \leq t \leq T-\delta$, and the proof is complete. 
The first statement of Theorem 3.1 now follows from Lemma 3.2 with $g=f$.

b) The second statement is a direct consequence of the first one. Indeed, from the hypothesis and a) it follows that $\operatorname{supp}(f) \subseteq[0, \delta]$ for all $\delta>0$. Hence $f \equiv 0$, and therefore $\alpha_{m}=0$ for all $m \geq 1$.

c) Let us now prove the third statement, i.e. the existence of exponentially growing coefficients $\alpha_{m}$ for which (3.22) admits a solution. Consider for instance the function

$$
F(z)=\frac{\sin (i \delta z)}{i \delta z} .
$$

Obviously, $F$ is an entire function that is bounded along the imaginary axis, satisfies

$$
\int_{-\infty}^{\infty}|F(i y)|^{2} d y<\infty
$$

and, moreover, is of exponential type $=\delta$.

According to Proposition 3.3, the moment problem (3.21) has a solution in the interval $(0, S)$ with $S \geq 2 \delta$ if $\left\{\alpha_{m}\right\}_{m \geq 1}$ are such that $F(m)=\alpha_{m} e^{-m S / 2}$. In view of (3.34) this is equivalent to

$$
\alpha_{m}=\frac{\sin (i \delta m)}{i \delta m} e^{m S / 2}
$$

Obviously,

$$
\left|\alpha_{m}\right| \sim \frac{C}{m} e^{m(S / 2+\delta)}, \quad m \rightarrow \infty .
$$

This completes the proof of Theorem 3.1

Proof of Theorem 3.2. To prove Theorem 3.2 it is sufficient to apply Theorem 3.1 with

$$
\alpha_{m}=\frac{a_{m}}{\phi_{m}^{\prime}(0)} .
$$

We now need an estimate on $\phi_{m}^{\prime}(0)$ as $m \rightarrow \infty$.

Lemma 3.3. If $\phi_{m}$ are the eigenfunctions described in section 2.3, then

$$
\left|\phi_{m}^{\prime}(0)\right| \sim \frac{1}{\sqrt{\pi}} \sqrt[4]{m} \text { as } m \rightarrow \infty .
$$

Proof. As described in section 2.3, the eigenfunctions $\phi_{m}$ are of the form

$$
\phi_{m}(y)=C_{m} \varphi_{2 m}(y)=C_{m} \frac{d^{2 m-1}\left[e^{-y^{2} / 4}\right]}{d y^{2 m-1}}
$$

with $C_{m}>0$ such that

$$
\left\|\phi_{m}\right\|_{L^{2}\left(\mathbb{R}_{+} ; K\right)}=1
$$

Let us recall that the Hermite polynomials $H_{n}$ are defined as

$$
H_{n}(y)=(-1)^{n} e^{y^{2}} \frac{d^{n}\left[e^{-y^{2}}\right]}{d y^{n}}, n \geq 0 .
$$

Therefore

$$
\phi_{m}(y)=-\frac{C_{m}}{2^{2 m-1}} H_{2 m-1}(y / 2) e^{-y^{2} / 4}
$$


It is well known that (see [20])

$$
\int_{-\infty}^{\infty} H_{n}(y) H_{k}(y) e^{-y^{2}} d y=2^{n} n ! \sqrt{\pi} \delta_{n k} .
$$

Consequently,

$$
\begin{aligned}
\| \phi_{m} & \|_{L^{2}\left(\mathbb{R}_{+}, K\right)}^{2}=\frac{C_{m}^{2}}{2^{4 m-2}} \int_{0}^{\infty}\left|H_{2 m-1}(y / 2)\right|^{2} e^{-y^{2} / 4} d y \\
& =\frac{C_{m}^{2}}{2^{4 m-2}} \int_{-\infty}^{\infty}\left|H_{2 m-1}(y)\right|^{2} e^{-y^{2}} d y=\frac{C_{m}^{2}(2 m-1) ! \sqrt{\pi}}{2^{2 m-1}} .
\end{aligned}
$$

Thus, in view of (3.40),

$$
C_{m}=\sqrt{\frac{2^{2 m-1}}{(2 m-1) ! \sqrt{\pi}}} .
$$

Moreover

$$
\phi_{m}^{\prime}(0)=-\frac{C_{m}}{2^{2 m}} H_{2 m-1}^{\prime}(0)
$$

But,

$$
H_{2 m-1}^{\prime}(0)=2(2 m-1) H_{2 m-2}(0)=(-1)^{m-1} \frac{2(2 m-1) !}{(m-1) !} .
$$

Consequently

$$
\phi_{m}^{\prime}(0)=\frac{(-1)^{m}}{\pi^{1 / 4}} \sqrt{\frac{(2 m-1) !}{[(m-1) !]^{2} 2^{2 m-1}}} .
$$

If we now apply Stirling's formula (see Olver [20])

$$
x ! \sim \sqrt{2 \pi x}\left(\frac{x}{e}\right)^{x} \text { as } x \rightarrow \infty,
$$

we deduce that

$$
\left|\phi_{m}^{\prime}(0)\right| \sim \frac{1}{\sqrt{\pi}}(m-1 / 2)^{1 / 4} \quad \text { as } m \rightarrow \infty
$$

and the proof of the lemma is complete.

This shows that, if the sequence of Fourier coefficients $\left\{a_{m}\right\}_{m \geq 1}$ satisfies the exponential growth condition (3.29), then $\alpha_{m}=a_{m} / \phi_{m}^{\prime}(0)$ satisfies (3.25) as well.

Theorem 3.2 is then a direct consequence of Theorem 3.1 .

3.4. On the lack of observability estimates. As we described in the introduction, a natural approach to the problem of null-controllability of heat equations is through the observability problem for the adjoint system (see, for instance, 7]).

More precisely, the null-controllability of system (3.3) with controls in $L^{2}(0, S)$ and for initial data in $L^{2}\left(\mathbb{R}_{+} ; K\right)$ is equivalent to the existence of a positive constant $C>0$ such that

$$
\|\xi(0)\|_{L^{2}\left(\mathbb{R}_{+}, K\right)}^{2} \leq C \int_{0}^{S}\left|\xi_{y}(0, S)\right|^{2} d s, \quad \forall \xi^{0} \in H_{0}^{1}\left(\mathbb{R}_{+} ; K\right),
$$

where, we recall, $\xi$ is the solution of (3.6)

As we have shown in Theorem [3.2 this null-controllability result is false and therefore (3.50) does not hold. In fact, according to Theorem 3.2 it turns out that 
all the possible weaker versions of (3.50) in which the $L^{2}$-norm of the left hand side is replaced by a $H^{-\sigma}$-norm for any $\sigma>0$ are false as well.

In this section we describe how the lack of observability inequalities of the form (3.50) may be obtained directly.

Let us recall that inequalities of the form (3.50) are well-known to be true in context of bounded domains.

In view of the Fourier series expansion of the solution $\xi$ of (3.6), we have

$$
\xi(y, s)=\sum_{m \geq 1} b_{m} e^{-(m-1 / 2)(S-s)} \phi_{m}(y) .
$$

Thus

$$
\xi_{y}(0, S)=\sum_{m \geq 1} b_{m} e^{-(m-1 / 2)(S-s)} \phi_{m}^{\prime}(0)
$$

Taking into account that, from Lemma 3.3, $\left|\phi_{m}^{\prime}(0)\right| \sim C m^{1 / 4}$ as $m \rightarrow \infty$, we deduce that (3.50) is equivalent to

$$
\begin{aligned}
\sum_{m \geq 1} \frac{a_{m}^{2}}{\sqrt{m}} e^{-(2 m-1) S} & \leq C \int_{0}^{S}\left|\sum_{m \geq 1} a_{m} e^{-(m-1 / 2)(S-s)}\right|^{2} d s \\
& =C \int_{0}^{S}\left|\sum_{m \geq 1} a_{m} e^{-(m-1 / 2) s}\right|^{2} d s,
\end{aligned}
$$

or, even, to

$$
\sum_{m \geq 1} \frac{a_{m}^{2}}{\sqrt{m}} e^{-2 m S} \leq C \int_{0}^{S}\left|\sum_{m \geq 1} a_{m} e^{-m s}\right|^{2} d s .
$$

Inequalities of the form (3.51) are well known to be true when the sequence of real exponentials $\left\{e^{-m s}\right\}_{m \geq 1}$ is replaced by $\left\{e^{-m^{\alpha} s}\right\}_{m \geq 1}$ with $\alpha>1$ (see, for instance, [5] and [22]). The case $\alpha=1$ we are considering is critical, since the series $\sum_{m \geq 1} 1 / m$ diverges.

In this critical case the following negative result holds:

Proposition 3.4. There is no sequence $\left\{\rho_{m}\right\}_{m \geq 1}$ of positive weights, i.e. $\rho_{m}>0$ for all $m \geq 1$, such that

$$
\sum_{m \geq 1} \rho_{m} a_{m}^{2} \leq \int_{0}^{S}\left|\sum_{m \geq 1} a_{m} e^{-m s}\right|^{2} d s
$$

for all $\left\{a_{m}\right\}$.

Remark 3.9. This result excludes the inequality (3.50) and any other weaker version of it. According to this result, the positive statement of Theorem 3.2 on the existence of exponentially growing Fourier coefficients leading to null-controllable initial data may not be obtained as a consequence of an observability inequality of the form (3.52). 
Observe that an inequality like (3.52) is, roughly speaking, equivalent to the null controllability in time $S$ of all initial data in the class

$$
H=\left\{u_{0}=\sum_{k \geq 1} a_{k} \phi_{k}: \sum_{k \geq 1} \frac{a_{k}^{2}}{\rho_{k}}<\infty\right\},
$$

and, according to Theorem 3.2 we know that this is false, whatever the sequence of weights $\left\{\rho_{k}\right\}$ is.

Proposition 3.4 is an immediate consequence of the following, one:

Proposition 3.5. Let $\left\{\lambda_{j}\right\}_{j \geq 1}$ be an increasing sequence of positive real numbers. Assume that there exists a sequence of positive weights $\left\{\rho_{j}\right\}_{j \geq 1}$ such that

$$
\sum_{j \geq 1} \rho_{j}\left|a_{j}\right|^{2} \leq \int_{0}^{1}\left|\sum_{j \geq 1} a_{j} e^{-\lambda_{j} t}\right|^{2} d t
$$

for all $\left\{a_{j}\right\}_{j \geq 1}$. Then, necessarily,

$$
\sum_{j \geq 1} \frac{1}{\lambda_{j}}<\infty
$$

Proof of Proposition 3.5. As a consequence of (3.53) we have

$$
\sum_{j \geq 1} \rho_{j} a_{j}^{2} \leq \int_{0}^{\infty}\left|\sum_{j \geq 1} a_{j} e^{-\lambda_{j} t}\right|^{2} d t,
$$

for all $\left\{a_{j}\right\}_{j \geq 1}$.

We now use the following result in [28], p. 151:

Let there be given $\left\{f_{j}\right\}_{j \geq 1}$ be a sequence in a Hilbert space $H$ and a sequence of real numbers $\left\{c_{j}\right\}_{j \geq 1}$ such that

$$
\left|\sum_{j=1}^{N} a_{j} c_{j}\right| \leq M\left\|\sum_{j=1}^{N} a_{j} f_{j}\right\|_{H}
$$

for any $\left\{a_{j}\right\}_{1 \leq j \leq N}$ and any $N \in \mathbb{N}$. Then, there exists $f \in H$ such that $\|f\|_{H} \leq M$ and $\left(f, f_{j}\right)=c_{j}, j \geq 1$.

We apply this result with $H=L^{2}(0, \infty)$, and $f_{j}=e^{-\lambda_{j} t}$. According to (3.55), (3.56) holds with $\left\{c_{j}\right\}$ such that, for some $j_{0}, c_{j}=0$ for all $j \neq j_{0}$, and $c_{j_{0}}=1$. We fix $j_{0}=1$ and consider the sequence

$$
c_{j}=0, \quad \forall j \geq 2 ; \quad c_{1}=1 .
$$

According to the above result we deduce the existence of $f \in L^{2}(0, \infty)$ such that

$$
\|f\|_{L^{2}(0, \infty)} \leq \frac{1}{\sqrt{\rho_{1}}} ; \int_{0}^{\infty} f(t) e^{-\lambda_{j} t} d t= \begin{cases}1, & j=1 \\ 0, & j \geq 2 .\end{cases}
$$

Hence, the exponential family $\left\{e^{-\lambda_{n} t}\right\}_{j \geq 2}$ is not total in $L^{2}(0, \infty)$. From Müntz's Theorem (see Schwartz [23], p. 24) we obtain

$$
\sum_{j \geq 2} \frac{1}{\lambda_{j}}<\infty
$$

and the proof is complete. 
Remark 3.10. The proof of Proposition 3.5] actually provides a stronger result. Namely, it shows that if the sequence $\left\{\lambda_{j}\right\}_{j \geq 1}$ is such that for some $j_{0}$ and $\rho>0$ we have

$$
\rho\left|a_{j_{0}}\right|^{2} \leq \int_{0}^{1}\left|\sum_{j \geq 0} a_{j} e^{-\lambda_{j} t}\right|^{2} d t
$$

for all sequences $\left\{a_{j}\right\}_{j \geq 1}$, then, necessarily,

$$
\sum_{j \geq 1} \frac{1}{\lambda_{j}}<\infty
$$

Inequalities of the form (3.57) are equivalent to what is called the spectral controllability problem. In this setting it consists in analyzing whether the eigenfunctions may be driven to zero in finite time. Theorem 3.2 provides a negative answer. Proposition 3.5 provides a second proof of this negative result, in which the effect of the divergence of the series $\Sigma 1 / \lambda_{j}$ is clearly seen.

\section{Further COMments AND OPEN PROBLEMS}

4.1. General domains. The problem of null-controllability of the heat equation arises in fact in any domain $\Omega$ of $\mathbb{R}^{n}$. As we have described in the introduction, when $\Omega$ is bounded and of class $C^{2}$ the null-controllability is well known. The results we have proved in the one-dimensional case can be used in the context of some simple unbounded domains.

For instance, if $\Omega=\mathbb{R}_{+}^{n}, n \geq 2$, and the control acts on the whole boundary $\partial \Omega$, the situation is similar to the one encountered in one space dimension. Namely:

a) Initial data with Fourier coefficients that grow less than any exponential are not null-controllable in any time.

b) There are exponentially growing Fourier coefficients that are null-controllable. This can be done by using separation of variables in order to reduce the problem to an infinite family of one-dimensional problems (see [19]).

The approach based on the use of the similarity variables may also be used in general conical domains. But, due to the lack of orthogonality of the traces of the normal derivatives of the eigenfunctions, the corresponding moment problem is more complex and remains to be solved.

When $\Omega$ is a general unbounded domain, the similarity transformation does not seem to be of any help, since the domain one gets after transformation depends on time.

Therefore, a completely different approach seems to be needed when $\Omega$ is not conical. However, one may still expect a bad behavior of the null-control problem. Indeed, assume for instance that $\Omega$ contains $\mathbb{R}_{+}^{n}$. If one is able to control to zero in $\Omega$ an initial datum $u_{0}$ by means of a boundary control acting on $\partial \Omega \times(0, T)$, then, by restriction, one is able to control the initial datum $\left.u_{0}\right|_{\mathbb{R}_{+}^{n}}$ with the control being the restriction of the solution in the larger domain $\Omega \times(0, T)$ to $\mathbb{R}^{n-1} \times(0, T)$. A careful development of this argument and of the result it may lead to remains to be done.

The approximate control problem for the semilinear heat equation in general unbounded domains was addressed in [26]. There, an approximation method was developed. The domain $\Omega$ was approximated by bounded domains (essentially by $\Omega \cap B_{R}, B_{R}$ being the ball of radius $R$ ) and the approximate control in the 
unbounded domain $\Omega$ was obtained as the limit of the approximate control on the approximating bounded domain $\Omega \cap B_{R}$.

However, this approach does not apply in the context of the null-control problem.

The approach described in [14] and [16] is also worth mentioning. It is proved that, for any $T>0$, the heat equation has a fundamental solution which is $C^{\infty}$ away from the origin and with support in the strip $0 \leq t \leq T$. This allows one to build a solution $u$ of the heat equation

$$
u_{t}-\Delta u=0 \quad \text { in } \mathbb{R}^{n} \times(0, T)
$$

that is continuous in $\mathbb{R}^{n} \times[0, T]$ and matches the initial and final conditions

$$
\begin{aligned}
& u(x, 0)=u_{0} \quad \text { in } \mathbb{R}^{n}, \\
& u(x, T)=0 \quad \text { in } \mathbb{R}^{n},
\end{aligned}
$$

for any continuous function $u_{0}$.

This may also be interpreted as a null-controllability result in a general domain $\Omega$. Indeed, by setting $v=\left.u\right|_{\partial \Omega \times(0, T)}$, we deduce that $\left.u\right|_{\Omega \times(0, T)}$, the restriction of $u$ solution of (4.1)-(4.3) to $\Omega \times(0, T)$, satisfies

$$
\begin{cases}u_{t}-\Delta u=0 & \text { in } \Omega \times(0, T), \\ u=v & \text { on } \partial \Omega \times(0, T), \\ u(x, 0)=u_{0}(x) & \text { in } \Omega, \\ u(x, T)=0 & \text { in } \Omega .\end{cases}
$$

This argument applies when $\Omega$ is unbounded as well. In particular, when $n=1$ and $\Omega=\mathbb{R}_{+}$, this shows that for any $u_{0} \in C\left(\mathbb{R}_{+}\right)$there exist $v \in C[0, T]$ and a solution $u$ of

$$
\begin{cases}u_{t}-u_{x x}=0, & 0<x, \\ u(0, t)=v(t), & 0<t<T, \\ u(x, 0)=u_{0}(x), & 0<x\end{cases}
$$

such that

$$
u(x, T)=0 \quad \text { in } \mathbb{R}_{+} .
$$

Note however that the solutions of (4.5)-(4.6) provided that the approach of [14] and [16] do not fit in the context of our negative result, since they grow too fast as $|x| \rightarrow \infty$, and therefore, these are not solutions in the sense of transposition.

It is also worth comparing this result with the positive one of Section 3 In Theorem 3.2 we prove that initial data with exponentially growing Fourier coefficients are null-controllable by means of $L^{2}(0, T)$ controls. Moreover, the trajectory we obtain necessarily has exponentially growing Fourier coefficients during the whole time interval. This is in agreement with the result of [14] and [16], in which the trajectory has also a fast growth rate as $|x| \rightarrow \infty$.

4.2. More general equation. The same problems arise in the context of more general parabolic equations, including variable coefficients, semilinear terms, ....

We refer to [6] and [8] for the analysis of the null-control problem of the semilinear heat equation in bounded domains, and to [12] for the case of linear heat equations with variable coefficients.

The approach we have adopted in this work does not seem to extend to these more general problems, even in $\mathbb{R}_{+}^{n}$, except for very particular cases. Indeed, Fourier 
series decompositions of the solutions apply for equations of the form

$$
w_{s}-\Delta w-\frac{y \cdot \nabla w}{2}-\frac{n}{2} w+a(y) w+b(s) w=0 .
$$

Obviously, in order to get an equation of the form (4.7) in the similarity variables, the original equation

$$
u_{t}-\Delta u+c(x, t) u=0
$$

needs to have a variable coefficient $c(x, t)$ of a very special structure.

The extension of the results of this paper in, say $\mathbb{R}_{+}^{n}$, to the general case of coefficients $c \in L^{\infty}\left(\mathbb{R}_{+}^{n} \times(0, T)\right)$ remains to be done.

4.3. Necessary and sufficient conditions for null-controllability. The results of this paper show that, when $\Omega=\mathbb{R}_{+}^{n}$,

$\star$ Initial data with Fourier coefficients growing slower than any exponential may not be controlled in finite time by means of controls in a suitable weighted $L^{2}$ space of the boundary.

$\star$ Some initial data with exponentially growing Fourier coefficients are controllable.

Of course, it would be desirable to obtain a more explicit characterization of the Fourier coefficients of the null-controllable initial data.

4.4. Approximation by means of domains of finite measure. As we mentioned above, a natural approach to the problem of null-controllability of the heat equation in an unbounded domain $\Omega$ consists of, first, approximating this domain by ounded domains $\Omega_{R}$, second, solving the null-control problem in $\Omega_{R}$, and finally, letting $R \rightarrow \infty$.

This was done successfully in 26 in the context of approximate controllability.

In this section we describe why this method fails in the context of the null-control problem, in agreement with the negative results we have proved in this paper.

Let us consider the one-dimensional problem discussed in Section 3 Then, $\Omega=$ $\mathbb{R}_{+}$. We then set $\Omega_{R}=(0, R)$. Consider the heat equation in $\Omega_{R}$ with control in the left end $x=0$, i.e.

$$
\begin{cases}u_{t}-u_{x x}=0, & 0<x<R, \quad 0<t<T, \\ u(0, t)=v(t), u(R, t)=0, & 0<t<T, \\ u(x, 0)=u_{0}(x), & 0<x<R .\end{cases}
$$

We assume, to simplify things, that $u_{0} \in \mathcal{D}\left(\mathbb{R}_{+}\right)$. Consequently, the support of $u_{0}$ is contained in $(0, R)$ for all $R>0$ sufficiently large.

We fix $T>0$.

It is by now well-known that this system is null-controllable. Thus, given $T>0$ and $u_{0}$ as above, for any $R>0$ there exists $v_{R} \in L^{2}(0, T)$ such that the solution $u_{R}$ of (4.9) satisfies

$$
u_{R}(x, T)=0, \quad 0<x<R .
$$

It is then natural to analyze whether the family $\left\{v_{R}\right\}$ remains bounded as $R \rightarrow$ $\infty$. If it were bounded, by weak convergence we would get a null-control $v \in$ $L^{2}(0, T)$ for $u_{0}$ in time $T$ in the half-line $(0, \infty)$. But, according to the negative results of Section 3 we know that null-controllability fails in $(0, \infty)$. Thus, the sequence $\left\{v_{R}\right\}$ has to diverge. In this section we briefly describe how this divergence may be seen to hold. 
The control of system (4.9) may be reduced to the problem of null-controllability of the heat equation in the interval $(0,1)$ but in a time interval that tends to zero as $R \rightarrow \infty$. More precisely, $u$ satisfies (4.9) if and only if

$$
z(x, t)=u\left(R x, R^{2} t\right)
$$

satisfies

$$
\begin{cases}z_{t}-z_{x x}=0, & 0<x<1,0<t<T R^{-2} \\ z(0, t)=v\left(R^{2} t\right), & 0<t<T R^{-2} \\ z(1, t)=0, & 0<t<T R^{-2} \\ z(x, 0)=u_{0}(R x), & 0<x<1 .\end{cases}
$$

Note that the control time in (4.12) is $T R^{-2}$, which tends to zero as $R \rightarrow \infty$.

As we mentioned above, the null-control for system 4.12 may be obtained through a suitable observability estimate for the adjoint system

$$
\begin{cases}\varphi_{t}+\varphi_{x x}=0, & 0<x<1,0<t<T R^{-2} \\ \varphi(0, t)=\varphi(1, t)=0, & 0<t<T R^{-2} \\ \varphi(x, T)=\varphi_{0}(x), & 0<x<1 .\end{cases}
$$

Indeed, let $C_{R}>0$ be the best positive constant of the observability inequality

$$
\|\varphi(0)\|_{L^{2}(0,1)}^{2} \leq C_{R} \int_{0}^{T R^{-2}}\left|\varphi_{x}(0, t)\right|^{2} d t .
$$

Then, the control of (4.12) admits the bound

$$
\left\|v\left(R^{2} t\right)\right\|_{L^{2}\left(0, T R^{-2}\right)} \leq \sqrt{C_{R}}\left\|u_{0}(R x)\right\|_{L^{2}(0,1)},
$$

or, in other words, the control $v_{R}$ of (4.9) satisfies

$$
\left\|v_{R}\right\|_{L^{2}(0, T)} \leq \sqrt{R C_{R}}\left\|u_{0}\right\|_{L^{2}(0, R)} .
$$

However, we know that the constant $C_{R}$ in 4.14 is of the order of $e^{C(T) R^{2}}$ as $R \rightarrow \infty$. And this estimate is sharp. This sharp estimate may be found in 11] and [24], where the problem is addressed analyzing the biorthogonal family associated to the sequence of real exponentials $\left\{e^{-j^{2} t}\right\}_{j \geq 1}$ in $L^{2}(0, \delta)$ as $\delta \rightarrow 0$. In 7] sharp observability estimates were proved by means of Carleman inequalities in the context of the internal control problem for general bounded domains in any space dimension. The same estimates apply for the boundary control problem.

The above developments show why the method on approximating the unbounded domain by bounded domains fails for the null-control problem.

4.5. A result of backwards uniqueness for the non-homogeneous heat equation. The main result of non-controllability from Theorem 3.1 can have the following different interpretation:

Theorem. Let $w^{j}(j=1,2)$ be two solutions of (3.3) on $Q=\mathbb{R}_{+} \times(0, S)$ with non-homogeneous terms $\widetilde{v}^{1}$ and $\widetilde{v}^{2}$ respectively in $L^{2}(0, S)$.

Suppose that the expansion coefficients $\left(\alpha_{m}^{j}\right)_{m \geq 1}$ of the initial data $w^{j}(\cdot, 0)$ satisfy

$$
\lim \sup \left\{\left[\log \left|\alpha_{m}^{i}\right|\right] / m\right\} \leq 0, \quad j=1,2 .
$$

Then equality of the terminal states $w^{1}(\cdot, S)=w^{2}(\cdot, S)$ implies $w^{1}=w^{2}$ in $Q$. 
Proof. Indeed, the solutions $w^{j}(j=1,2)$ can be written as

$$
w^{j}(\cdot, s)=\sum_{m \geq 1} \alpha_{m}^{j}(s) \phi_{m}(\cdot), \quad s \in[0, S],
$$

where $\alpha_{m}^{j}(0)=\alpha_{m}^{j}, m \geq 1$.

From the hypothesis we have that $w^{j}(\cdot, 0) \in \mathcal{X}_{\delta}^{*}$, for all $\delta>0$. The existence result from section 3.1 implies that $w^{j} \in C\left([0, S], \mathcal{X}_{\delta}^{*}\right)$, for all $\delta>0$. Therefore, for each $s \in[0, S]$,

$$
\lim \sup \left\{\left[\log \left|\alpha_{m}^{j}(s)\right|\right] / m\right\} \leq 0, \quad j=1,2 .
$$

Let us now define $w=w^{1}-w^{2}$.

The Fourier coefficients of $w$ are $\alpha_{m}(s)=\alpha_{m}^{1}(s)-\alpha_{m}^{2}(s), m \geq 1$, and satisfy (3.24). Moreover, $w(\cdot, S)=0$. Hence, for each $s \in[0, S], w(\cdot, s)$ is null-controllable. From Theorem 3.1 it follows that $\alpha_{m}(s)=0$ for all $m \geq 1$. Hence $w^{1}(\cdot, s)=w^{2}(\cdot, s)$ for all $s \in[0, S]$.

Let us remark that, in the case of a bounded interval $\Omega$, this backwards uniqueness result does not hold (it contradicts the well-known positive controllability result we have mentioned before).

On the other hand, our positive control result shows that the condition (4.16) on the Fourier coefficients is necessary in order to have this backwards uniqueness result for the non-homogeneous heat equation.

\section{REFERENCES}

[1] S.A. Avdonin and S.A. Ivanov, Families of exponentials. The method of moments in controllability problems for distributed parameter systems, Cambridge University Press, 1995. MR 97b:93002

[2] R.P. Boas, Entire functions, Academic Press, New York, 1954. MR 16:914f

[3] C. Bandle and H.A. Levine, On the existence and nonexistence of global solutions of reaction-diffusion equations in sectorial domains, Trans. Amer. Math. Soc., 316 (2) (1989), 595-622. MR 90c:35118

[4] M. Escobedo and O. Kavian, Variational problems related to self-similar solutions of the heat equation, Nonlinear Analysis TMA, 11 (10) (1987), 1103-1133. MR 90a:3512q

[5] H. Fattorini and D.L. Russell, Uniform bounds on biorthogonal functions for real exponentials with an application to the control theory of parabolic equations, Quarterly J. Appl. Math., 32 (1974), 45-69. MR 58:23325

[6] E. Fernández-Cara, Null controllability of the semilinear heat equation, ESAIM:COCV, 2 (1997), 87-107, (http://www.emath.fr/cocv/). MR 98d:93011

[7] E. Fernández-Cara and E. Zuazua, The cost of approximate controllability for heat equations: The linear case, Advances Diff. Eqs, 5 (2000), 465-514.

[8] E. Fernández-Cara and E. Zuazua, Null controllability of weakly blowing-up semilinear heat equations, preprint, 1999.

[9] A. Fursikov and O. Yu. Imanuvilov, Controllability of evolution equations, Lecture Notes Series \#34, Research Institute of Mathematics, Global Analysis Research Center, Seoul National University, 1996. MR 97g:93002

[10] J.M. Ghidaglia, Some backward uniqueness results, Nonlinear Anal. TMA, 10 (1986), 777790. MR 87m:34083

[11] E. N. Güichal, A lower bound on the norm of the control operator for the heat equation, J. Math. Anal. Appl., 110 (1985), 519-527. MR 87i:93069

[12] O. Yu. Imanuvilov and M. Yamamoto, On Carleman inequalities for parabolic equations in Sobolev spaces of negative order and exact controllability for semilinear parabolic equations, Preprint n 98-46, University of Tokyo, Graduate School of Mathematics, Japan, 1998.

[13] F. John, Partial Differential Equations, Springer Verlag, 1978. MR 87g:35002 (later ed.) 
[14] B.F. Jones, Jr., A fundamental solution of the heat equation which is supported in a strip, J. Math. Anal. Appl., 60 (1977), 314-324. MR 56:9070

[15] O. Kavian, Remarks on the large time behavior of a nonlinear diffusion equation, Ann. Inst. Henri Poincaré, Analyse non linéaire, 4 (5) (1987), 432-452. MR 89b:35013

[16] W. Littman, Boundary control theory for hyperbolic and parabolic partial differential equations with constant coefficients, Annali Scuola Norm. Sup. Pisa, Serie IV, 3 (1978), 567-580. MR 80a:35023

[17] J.L. Lions and B. Malgrange, Sur l'unicité rétrograde dans les problèmes mixtes paraboliques, Math. Scan., 8 (1960), 277-286. MR 25:4269

[18] G. Lebeau and L. Robbiano, Contrôle exact de l'équation de la chaleur, Comm. P.D.E., 20 (1995), 335-356. MR 95m:93045

[19] S. Micu and E. Zuazua, On the lack of null-controllability of the heat equation on the half space, Portugal. Math., to appear.

[20] F.W.J. Olver, Asymptotics and special functions, Academic Press, 1974. MR 55:8655

[21] L. Rosier, Exact controllability for the linear Korteweg-de Vries equation on the half-line, SIAM J. Cont. Optim., 39 (2000), 331-351.

[22] D.L. Russell, Controllability and stabilizability theory for linear partial differential equations. Recent progress and open questions, SIAM Rev., 20 (1978), 639-739. MR 80c:93032

[23] L. Schwartz, Etude des sommes d'exponentielles, Hermann, Paris, 1959. MR 21:5116

[24] T.I. Seidman, S.A. Avdonin and S.A. Ivanov, The "window problem" for series of complex exponentials, preprint, 1998.

[25] L. de Teresa, Approximate controllability of a semilinear heat equation in $\mathbb{R}^{N}$, SIAM J. Cont. Optim., 36 (6) (1996), 855-884. MR 99j:93011

[26] L. de Teresa and E. Zuazua, Approximate controllability of the heat equation in unbounded domains, Nonlinear Anal. TMA, 37 (1999), 1059-1090. CMP 99:14

[27] K. Yosida, Functional Analisis, Springer Verlag, 1980. MR 82i:46002

[28] R.M. Young, An introduction to nonharmonic Fourier series, Academic Press, 1980. MR 81m:42027

Departamento de Matemática Aplicada, Universidad Complutense, 28040 Madrid, SPAIN

E-mail address: sorin@sunma4.mat.ucm.es

Departamento de Matemática Aplicada, Universidad Complutense, 28040 Madrid, SPAIN

E-mail address: zuazua@eucmax.sim.ucm.es 